\title{
Singular value decomposition versus sparse grids: \\ Refined complexity estimates
}

M. Griebel and H. Harbrecht

Departement Mathematik und Informatik

Preprint No. 2017-08

Fachbereich Mathematik

July 2017

Universität Basel

CH-4051 Basel

www.math.unibas.ch 
IMA Journal of Numerical Analysis (2017) Page 1 of 17

doi:10.1093/imanum/drnxxx

\title{
Singular value decomposition versus sparse grids: Refined complexity estimates
}

\author{
MICHAEL GRIEBEL $\dagger$ \\ Institut für Numerische Simulation, Universität Bonn, Wegelerstr. 6, 53115 Bonn, Germany \\ and \\ Fraunhofer Institute for Algorithms and Scientific Computing (SCAI), Schloss Birlinghoven, \\ 53754 Sankt Augustin, Germany \\ AND \\ HELMUT HARBRECHT $\$$ \\ Departement Mathematik und Informatik, Universität Basel, Spiegelgasse 1, 4051 Basel, \\ Switzerland
}

[Received on 11 July 2017]

\begin{abstract}
We compare the cost complexities of two approximation schemes for functions which live on the product domain $\Omega_{1} \times \Omega_{2}$ of sufficiently smooth domains $\Omega_{1} \subset \mathbb{R}^{n_{1}}$ and $\Omega_{2} \subset \mathbb{R}^{n_{2}}$, namely the singular value / Karhunen-Lòeve decomposition and the sparse grid representation. We assume that appropriate finite element methods with associated orders $r_{1}$ and $r_{2}$ of accuracy are given on the domains $\Omega_{1}$ and $\Omega_{2}$, respectively. This setting reflects practical needs, since often black-box solvers are used in numerical simulation which restrict the freedom in the choice of the underlying discretization. We compare the cost complexities of the associated singular value decomposition and the associated sparse grid approximation. It turns out that, in this situation, the approximation by the sparse grid is always equal or superior to the approximation by the singular value decomposition. The results in this article improve and generalize those from Griebel \& Harbrecht (2014). We now especially consider the approximation of functions from generalized isotropic and anisotropic Sobolev spaces.
\end{abstract}

Keywords: singular value decomposition; sparse grids; complexity.

\section{Introduction}

With this article, we intend to refine the results which have been achieved in Griebel \& Harbrecht (2014), where we were concerned with the comparison of low-rank approximation methods and sparse grid methods for bivariate functions. This is a relevant setting since many problems in science and engineering lead to problems on the product $\Omega_{1} \times \Omega_{2}$ of two domains $\Omega_{1} \subset \mathbb{R}^{n_{1}}$ and $\Omega_{2} \subset \mathbb{R}^{n_{2}}$. For example, radiosity models and radiative transfer (Widmer et al. (2008)), space-time formulations of parabolic problems (Griebel \& Oeltz (2007)), phase space problems (Balescu (1997)), biscale homogenization (Cioranescu et al. (2008)), as well as correlation equations (Deb et al. (2001)) fit into this setting. We refer the reader to Griebel \& Harbrecht (2014) for a more comprehensive discussion of these problems and further references.

\footnotetext{
† griebel@ins.uni-bonn.de

*helmut.harbrecht@unibas.ch
}

(c) The author 2017. Published by Oxford University Press on behalf of the Institute of Mathematics and its Applications. All rights reserved. 
Moreover, the representation of functions on product domains by low-rank approximation is also the fundamental idea of reduced basis methods and model order reduction, see Hesthaven et al. (2016), Quarteroni et al. (2016), Rozza et al. (2008), and the references therein. Similarly, in uncertainty quantification, the spatial variable and the stochastic variable are defined on different domains. In general, after inserting the Karhunen-Lòeve decomposition of the underlying random field, one arrives at a parametric problem posed on the product of the physical domain and a high- or even infinite-dimensional parameter domain, see Ghanem \& Spanos (1991) and Le Maître \& Knio (2010) for example.

All the aforementioned problems are directly given on the product of two domains. Furthermore, for some of these as well as for many other problems, the domains themselves are products of lowerdimensional domains. Then, the domain of an $n$-dimensional problem with, for instance, $n$ being some power of two can be split into the product of two domains of dimension $n / 2$ which can recursively be further split until a terminal situation (a one-dimensional domain or a truly higher dimensional but nontensor product domain) is reached. Related representation methods have been considered in Bebendorf (2011), Grasedyck (2010), Hackbusch (2012), Hackbusch \& Kühn (2009), or Oseledets \& Tyrtyshnikov (2009). Here, one should note that hierarchical tensor formats, such as the hierarchical singular value decomposition or the tensor train format, exploit a truncated singular value decomposition for each dimension separation step. An alternative approach would here be a two-dimensional sparse grid approximation in each separation step. Then, the recursive application would yield an $n$-dimensional sparse grid. This motivates to consider the simple case of two domains $\Omega_{1}$ and $\Omega_{2}$ only. Our analysis covers then also a single bisection step in the above mentioned recursion.

Our setting is as follows. We suppose to have given, fixed sequences of nested trial spaces

$$
V_{0}^{(i)} \subset V_{1}^{(i)} \subset V_{2}^{(i)} \subset \cdots \subset L^{2}\left(\Omega_{i}\right), \quad i=1,2,
$$

on the individual subdomains, which consist of ansatz functions of approximation orders $r_{1}$ and $r_{2}$, respectively. We hence first fix the discretization and then compare the resulting algorithms. This reflects practical needs, since often black-box codes have to be used due to the implementational complexity of the underlying problems. Note at this point that our assumption is thus fundamentally different to the setting in approximation theory, where a function class is fixed and the best algorithm is sought, compare Novak \& Woźniakowski (2008), Novak \& Woźniakowski (2010), and Novak \& Woźniakowski (2012). It also different to the universality point of view, where one aims at algorithms, which are almost optimal for a wide range of function classes, see Babuška (1968) and Motornyj (1974) for example.

Having the trial spaces (1.1) at hand, we can either apply the truncated singular value decomposition

$$
f_{M}(\mathbf{x}, \mathbf{y}):=\sum_{\ell=1}^{M} \sqrt{\lambda_{\ell}} \varphi_{\ell}(\mathbf{x}) \psi_{\ell}(\mathbf{y}), \quad \mathbf{x} \in \Omega_{1}, \quad \mathbf{y} \in \Omega_{2}
$$

or the generalized sparse grid approach

$$
\widehat{f}_{J}(\mathbf{x}, \mathbf{y}):=\sum_{j_{1} / \sigma+j_{2} \sigma \leqslant J} \sum_{k_{1} \in \nabla_{j_{1}}^{(1)}} \sum_{k_{2} \in \nabla_{j_{2}}^{(2)}} \beta_{\left(j_{1}, k_{1}\right),\left(j_{2}, k_{2}\right)} \xi_{j_{1}, k_{1}}^{(1)}(\mathbf{x}) \xi_{j_{2}, k_{2}}^{(2)}(\mathbf{y}), \quad \mathbf{x} \in \Omega_{1}, \quad \mathbf{y} \in \Omega_{2}
$$

to represent a given function $f \in L^{2}\left(\Omega_{1} \times \Omega_{2}\right)$ in an efficient way. In the first representation, $\left\{\varphi_{\ell}\right\}_{\ell=1}^{M}$ and $\left\{\psi_{\ell}\right\}_{\ell=1}^{M}$ are sets of orthonormal functions. They are a-priorily unknown, can in general not be derived analytically, and need thus to be approximated in the ansatz spaces $\left\{V_{j}^{(i)}\right\}$. In other words, the approximation involves in most applications both, a truncation after $M$ terms and an approximate 
computation of the singular values and the associated left and right singular vectors. In the second representation, $\sigma>0$ is an appropriately chosen parameter and $\left\{\xi_{j, k}^{(i)}\right\}_{k \in \nabla_{j}^{(i)}, j \in \mathbb{N}}$ are in general multilevel or wavelet bases associated with the trial spaces, where the index $j$ refers to the level of resolution and the index $k$ refers to the locality of the basis function (the precise definition will be given in Section 4 ). In order to decide which approximation should be implemented for treating problems on product domains, we need to know the pro's and con's of both methods.

The main improvement of our theory in comparison to Griebel \& Harbrecht (2014) concerns the approximative truncated singular value decomposition. Namely, it turned out that it is not optimal to directly approximate the singular values and the eigenfunctions of the function under consideration. In this article, we therefore proceed differently: We first apply an $L^{2}$-projection on an appropriately chosen full tensor product space and then truncate the discrete singular value decomposition afterwards. This way, we are now able to directly derive sharp error estimates in the trace norm, while an approximation of the continuous eigenfunctions is no longer needed.

For our comparison, we consider the smoothness of the function $f$ to be measured in isotropic and anisotropic Sobolev norms. We then want to compare the cost complexity to reach an approximation with a prescribed accuracy for the truncated singular value decomposition and the sparse grid approach. One result of this article is then as follows: Given a function $f \in H^{p}\left(\Omega_{1} \times \Omega_{2}\right)$, we have to spend $\mathscr{O}\left(\varepsilon^{q}\right)$ degrees of freedom with

$$
q_{s v d}=\frac{\min \left\{n_{1}, n_{2}\right\}}{p}+\max \left\{\frac{\max \left\{n_{1}, n_{2}\right\}}{p}, \frac{n_{1}}{r_{1}}, \frac{n_{2}}{r_{2}}\right\}
$$

for the approximation by the truncated singular value decomposition and with

$$
q_{s g}=\max \left\{\frac{n_{1}+n_{2}}{p}, \frac{n_{1}}{r_{1}}, \frac{n_{2}}{r_{2}}\right\}
$$

for the general sparse grid method with associated parameter $\sigma=n_{1} / n_{2}$ (a precise definition is given in Section 4), see also Griebel \& Harbrecht (2013). Since it always holds

$$
\frac{\min \left\{n_{1}, n_{2}\right\}}{p}+\max \left\{\frac{\max \left\{n_{1}, n_{2}\right\}}{p}, \frac{n_{1}}{r_{1}}, \frac{n_{2}}{r_{2}}\right\} \geqslant \max \left\{\frac{n_{1}+n_{2}}{p}, \frac{n_{1}}{r_{1}}, \frac{n_{2}}{r_{2}}\right\},
$$

we deduce that the approximation by the sparse grid method is superior to the approximation by the singular value decomposition, at least for our setting. Moreover, we refine this result by considering in Section 5 more general isotropic and anisotropic Sobolev spaces. Also in these cases, the superiority of the sparse grid approach can be established. Recall here again that we fixed the underlying discretization via (1.1) and now compare the resulting associated algorithms.

The remainder of this article is organized as follows: In Section 2, we give a short introduction to multilevel approximation. In Section 3, we describe the singular value decomposition of a bivariate function on $\Omega_{1} \times \Omega_{2}$ and discuss its approximation properties in detail. Section 4 gives the basics of the so-called general sparse grid approximation of a bivariate function on $\Omega_{1} \times \Omega_{2}$ and presents its error rates and cost complexities. In Section 5, we compare the two approximations and make some final remarks.

Throughout this article, the notion "essential" in connection with the complexity estimates means "up to logarithmic terms". Moreover, to avoid the repeated use of generic but unspecified constants, we denote by $C \lesssim D$ that $C$ is bounded by a multiple of $D$ independently of parameters which $C$ and $D$ may depend on. Obviously, $C \gtrsim D$ is defined as $D \lesssim C$, and $C \sim D$ as $C \lesssim D$ and $C \gtrsim D$. 


\section{Preliminaries}

\subsection{Approximation on the subdomains}

Let $\Omega \subset \mathbb{R}^{n}$ be a sufficiently smooth, bounded domain. In general, one uses finite elements to approximate functions on $L^{2}(\Omega)$. In the present article, we focus on the common $h$-method, i.e., on finite elements of fixed approximation order. Then, particularly for applying multiscale techniques, one has a sequence of nested trial spaces

$$
V_{0} \subset V_{1} \subset V_{2} \subset \cdots \subset L^{2}(\Omega)
$$

such that

$$
L^{2}(\Omega)=\overline{\bigcup_{j \in \mathbb{N}_{0}} V_{j}}
$$

which is called multiscale analysis. Each space $V_{j}$ is defined by a single scale basis $\Phi_{j}=\left\{\phi_{j, k}\right\}$, i.e. $V_{j}=\operatorname{span}\left\{\phi_{j, k}: k \in \Delta_{j}\right\}$, where $\Delta_{j}$ denotes a suitable index set with cardinality $\# \Delta_{j} \sim 2^{n j}$.

We say that the trial spaces have (approximation) order $r \in \mathbb{N}$ if

$$
r=\sup \left\{s \in \mathbb{R}: \inf _{v_{j} \in V_{j}}\left\|v-v_{j}\right\|_{L^{2}(\Omega)} \lesssim h_{j}^{s}\|v\|_{s} \text { for all } v \in H^{s}(\Omega)\right\},
$$

where the quantity $h_{j} \sim 2^{-j}$ corresponds to the mesh width associated with the subspace $V_{j}$ on $\Omega$. Note that the integer $r>0$ refers in general to the maximal order of polynomials which are locally contained in $V_{j}$.

Equation (2.2) implies that a given function $v \in H^{p}(\Omega), 0 \leqslant p \leqslant r$, can be approximated in $V_{j}$ at a rate $h_{j}^{p}$, i.e., the associated $L^{2}$-orthogonal projection $Q_{j}: L^{2}(\Omega) \rightarrow V_{j}$ satisfies

$$
\left\|\left(I-Q_{j}\right) v\right\|_{L^{2}(\Omega)} \lesssim h_{j}^{p}\|v\|_{H^{p}(\Omega)}, \quad 0 \leqslant p \leqslant r .
$$

Thus, when we approximate a function $v \in H^{p}(\Omega)$ with $0 \leqslant p \leqslant r$ by uniform mesh refinement we obtain the rate $h_{j}^{p}$ according to (2.3). Since the mesh size and the number of unknowns in $V_{j}$ are related by $\operatorname{dim}\left(V_{j}\right) \sim 2^{j n} \sim h_{j}^{-n}$, we deduce that

$$
N \sim \varepsilon^{-n / p}
$$

unknowns have to be spent to achieve an approximation error $\varepsilon$. The best possible rate $N^{-n / r}$ is achieved if $p=r$, that is if $v \in H^{r}(\Omega)$.

\subsection{Kolmogorov's $n$-width and full tensor product spaces}

For our subsequent analysis of the approximation of bivariate functions in $L^{2}\left(\Omega_{1} \times \Omega_{2}\right)$, we shall fix the definitions, properties and cost complexities individually for each subdomain $\Omega_{i} \in \mathbb{R}^{n_{i}}, i=1,2$. That is, we fix two multiscale analyses

$$
V_{0}^{(i)} \subset V_{1}^{(i)} \subset V_{2}^{(i)} \subset \cdots \subset L^{2}\left(\Omega_{i}\right), \quad i=1,2,
$$

which are assumed to provide the approximation orders $r_{1}$ and $r_{2}$, respectively.

We start our discussion with the approximation of a given bivariate function $f \in L^{2}\left(\Omega_{1} \times \Omega_{2}\right)$ in full tensor product spaces $V_{j_{1}}^{(1)} \otimes V_{j_{2}}^{(2)}$. To measure the smoothness of bivariate functions, we define the Sobolev space of dominating mixed derivatives by

$$
H_{\text {mix }}^{s_{1}, s_{2}}\left(\Omega_{1} \times \Omega_{2}\right):=H^{s_{1}}\left(\Omega_{1}\right) \otimes H^{s_{2}}\left(\Omega_{2}\right)
$$


and set

$$
H_{i s o}^{s_{1}, s_{2}}\left(\Omega_{1} \times \Omega_{2}\right):=H_{\text {mix }}^{s_{1}, 0}\left(\Omega_{1} \times \Omega_{2}\right) \cap H_{\text {mix }}^{0, s_{2}}\left(\Omega_{1} \times \Omega_{2}\right) .
$$

Note that, in case of $p=p_{1}=p_{2}$, the space $H_{i s o}^{p, p}\left(\Omega_{1} \times \Omega_{2}\right)$ coincides with the standard isotropic Sobolev space $H^{p}\left(\Omega_{1} \times \Omega_{2}\right)$, i.e., it holds

$$
H^{p}\left(\Omega_{1} \times \Omega_{2}\right)=H_{i s o}^{p, p}\left(\Omega_{1} \times \Omega_{2}\right) .
$$

Let $f \in H_{i s o}^{p_{1}, p_{2}}\left(\Omega_{1} \times \Omega_{2}\right)$. Then, for the $L^{2}$-orthogonal projections onto $V_{j_{1}}^{(1)}$ and $V_{j_{2}}^{(2)}$, respectively, we obtain

$$
\begin{aligned}
&\left\|\left(I-Q_{j_{1}}^{(1)} \otimes I\right) f\right\|_{L^{2}\left(\Omega_{1} \times \Omega_{2}\right)} \lesssim 2^{-j_{1} \min \left\{p_{1}, r_{1}\right\}}\|f\|_{H_{\operatorname{mix}}^{\min \left\{p_{1}, r_{1}\right\}, 0}\left(\Omega_{1} \times \Omega_{2}\right)}, \\
&\left\|\left(I-I \otimes Q_{j_{2}}^{(2)}\right) f\right\|_{L^{2}\left(\Omega_{1} \times \Omega_{2}\right)} \lesssim 2^{-j_{2} \min \left\{p_{2}, r_{2}\right\}}\|f\|_{H_{\text {mix }}^{0, \min \left\{p_{2}, r_{2}\right\}}\left(\Omega_{1} \times \Omega_{2}\right)} .
\end{aligned}
$$

Using standard tensor product arguments leads thus to

$$
\begin{aligned}
\|(I- & \left.Q_{j_{1}}^{(1)} \otimes Q_{j_{2}}^{(2)}\right) f \|_{L^{2}\left(\Omega_{1} \times \Omega_{2}\right)} \\
& \lesssim 2^{-j_{1} \min \left\{p_{1}, r_{1}\right\}}\|f\|_{H_{\operatorname{mix}}^{\min \left\{p_{1}, r_{1}\right\}, 0}\left(\Omega_{1} \times \Omega_{2}\right)}+2^{-j_{2} \min \left\{p_{2}, r_{2}\right\}}\|f\|_{H_{\operatorname{mix}}^{0, \min \left\{p_{2}, r_{2}\right\}}\left(\Omega_{1} \times \Omega_{2}\right)} \\
& \lesssim\left(2^{-j_{1} \min \left\{p_{1}, r_{1}\right\}}+2^{-j_{2} \min \left\{p_{2}, r_{2}\right\}}\right)\|f\|_{H_{i s o}^{\min \left\{p_{1}, r_{1}\right\}, \min \left\{p_{2}, r_{2}\right\}}\left(\Omega_{1} \times \Omega_{2}\right)} .
\end{aligned}
$$

The optimum choice is to equilibrate the errors, since the approximation errors are additive while the cost are multiplicative. This means that

$$
j_{1} \min \left\{p_{1}, r_{1}\right\} \sim j_{2} \min \left\{p_{2}, r_{2}\right\},
$$

which implies, in view of $\operatorname{dim}\left(V_{j_{1}}^{(1)}\right) \sim 2^{j_{1} n_{1}}$ and $\operatorname{dim}\left(V_{j_{2}}^{(2)}\right) \sim 2^{j_{2} n_{2}}$, the cost complexity

$$
\operatorname{dof}_{f g}(\varepsilon)=\varepsilon^{-\frac{n_{1}}{\min \left\{p_{1}, r_{1}\right\}}} \varepsilon^{-\frac{n_{2}}{\min \left\{p_{2}, r_{2}\right\}}}
$$

to achieve a desired approximation error $\varepsilon$. If $p_{1} \leqslant r_{1}$ and $p_{2} \leqslant r_{2}$, this is known to be Kolmogorov's $n$-width for Sobolev balls in the space $H_{i s o}^{p_{1}, p_{2}}\left(\Omega_{1} \times \Omega_{2}\right)$, see Kolmogorov (1936). Hence, the cost complexity (2.8) is sharp in this case, which means, there is no better representation possible. Nonetheless, if $p_{1}>r_{1}$ or $p_{2}>r_{2}$, then (2.8) is not sharp anymore and we can approximate better in $H_{i s o}^{p_{1}, p_{2}}\left(\Omega_{1} \times \Omega_{2}\right)$ than by just using the full tensor product space.

The methods we discuss in this article are the approximative truncated singular value decomposition in Section 3 and the sparse grid in Section 4. The question we address is as follows: Given a function $H_{i s o}^{p_{1}, p_{2}}\left(\Omega_{1} \times \Omega_{2}\right)$, where $p_{1}, p_{2}>0$ are arbitrary and where trial spaces with approximation orders $r_{1}$ and $r_{2}$, respectively, are used in both approaches, which algorithm provides the cheaper approximation?

\section{Singular value decomposition}

\subsection{Definition and mapping properties}

We intend to numerically represent functions $f \in L^{2}\left(\Omega_{1} \times \Omega_{2}\right)$ on tensor product domains $\Omega_{1} \times \Omega_{2}$ in an efficient way. One way to solve this approximation problem is to use an ansatz by means of tensor products which separates the variables $\mathbf{x} \in \Omega_{1}$ and $\mathbf{y} \in \Omega_{2}$. We first consider the approximation

$$
f(\mathbf{x}, \mathbf{y}) \approx f_{M}(\mathbf{x}, \mathbf{y})=\sum_{\ell=1}^{M} \alpha_{\ell} \varphi_{\ell}(\mathbf{x}) \psi_{\ell}(\mathbf{y})
$$


with certain coefficients $\alpha_{\ell} \in \mathbb{R}$ and normalized functions $\varphi_{\ell} \in L^{2}\left(\Omega_{1}\right)$ and $\psi_{\ell} \in L^{2}\left(\Omega_{2}\right)$. Such an approximation is called low-rank approximation.

It is well known (see e.g. Ghanem \& Spanos (1991) or Lòeve (1978)) that, with respect to the number $M$ of terms, the best possible representation of a function $f \in L^{2}\left(\Omega_{1} \times \Omega_{2}\right)$ in the $L^{2}$-sense is given by the Karhunen-Lòeve / singular value decomposition. Then, $\alpha_{\ell}=\sqrt{\lambda_{\ell}}$ are given by the eigenvalues of the below defined integral operator (3.2) with kernel (3.3). As shown in Subsection 3.2, the truncation error (in terms of $M$ ) of the series (3.1) is related to the smoothness of the function $f$ to be approximated. As a byproduct of this estimate, we can infer the decay of the eigenvalues in Subsection 3.3. In Subsection 3.4, we finally consider the numerical treatment of (3.1). Besides determining the coefficients $\left\{\alpha_{\ell}\right\}_{\ell \in \mathbb{N}}$, a numerical scheme needs to approximate the functions $\left\{\varphi_{\ell}\right\}_{\ell \in \mathbb{N}}$ and $\left\{\psi_{\ell}\right\}_{\ell \in \mathbb{N}}$ in appropriate trial spaces $V_{j_{1}}^{(1)}$ and $V_{j_{2}}^{(2)}$, respectively, up to an accuracy corresponding to that of (3.1). Recall that the trial spaces which we consider are elements of the multiscale analyses (2.5) which have the approximation orders $r_{1}$ and $r_{2}$, respectively.

To derive the singular value decomposition, we shall consider the integral operator

$$
\mathscr{S}: L^{2}\left(\Omega_{1}\right) \rightarrow L^{2}\left(\Omega_{2}\right), \quad u \mapsto(\mathscr{S} u)(\mathbf{y}):=\int_{\Omega_{1}} f(\mathbf{x}, \mathbf{y}) u(\mathbf{x}) \mathrm{d} \mathbf{x} .
$$

Its adjoint is

$$
\mathscr{S}^{\star}: L^{2}\left(\Omega_{2}\right) \rightarrow L^{2}\left(\Omega_{1}\right), \quad u \mapsto\left(\mathscr{S}^{\star} u\right)(\mathbf{x}):=\int_{\Omega_{2}} f(\mathbf{x}, \mathbf{y}) u(\mathbf{y}) \mathrm{d} \mathbf{y} .
$$

To obtain the low-rank representation (3.1), we need to compute the eigenvalues of the integral operator

$$
\mathscr{K}=\mathscr{S}^{\star} \mathscr{S}: L^{2}\left(\Omega_{1}\right) \rightarrow L^{2}\left(\Omega_{1}\right), \quad u \mapsto(\mathscr{K} u)(\mathbf{x}):=\int_{\Omega_{1}} k\left(\mathbf{x}, \mathbf{x}^{\prime}\right) u\left(\mathbf{x}^{\prime}\right) \mathrm{d} \mathbf{x}^{\prime}
$$

whose kernel function is given by

$$
k\left(\mathbf{x}, \mathbf{x}^{\prime}\right)=\int_{\Omega_{2}} f(\mathbf{x}, \mathbf{y}) f\left(\mathbf{x}^{\prime}, \mathbf{y}\right) \mathrm{d} \mathbf{y} \in L^{2}\left(\Omega_{1} \times \Omega_{1}\right) .
$$

This is a Hilbert-Schmidt kernel. Thus, the associated integral operator $\mathscr{K}$ is compact. Moreover, since $\mathscr{K}$ is self-adjoint, there exists a decomposition into eigenpairs $\left(\lambda_{\ell}, \varphi_{\ell}\right)$, i.e.,

$$
\mathscr{K} \varphi_{\ell}=\lambda_{\ell} \varphi_{\ell} \quad \text { for all } \ell \in \mathbb{N},
$$

with non-negative eigenvalues $\lambda_{1} \geqslant \lambda_{2} \geqslant \cdots \geqslant \lambda_{m} \rightarrow 0$ and eigenfunctions $\left\{\varphi_{\ell}\right\}_{\ell \in \mathbb{N}}$, which constitute an orthonormal basis in $L^{2}\left(\Omega_{1}\right)$.

We now define for all $\ell \in \mathbb{N}$ with $\lambda_{\ell}>0$ the function $\psi_{\ell} \in L^{2}\left(\Omega_{2}\right)$ by

$$
\psi_{\ell}(\mathbf{y})=\frac{1}{\sqrt{\lambda_{\ell}}}\left(\mathscr{S} \varphi_{\ell}\right)(\mathbf{y})=\frac{1}{\sqrt{\lambda_{\ell}}} \int_{\Omega_{1}} f(\mathbf{x}, \mathbf{y}) \varphi_{\ell}(\mathbf{x}) \mathrm{d} \mathbf{x} .
$$

This constitutes a second sequence of orthonormal functions since

$$
\begin{aligned}
\left(\psi_{k}, \psi_{\ell}\right)_{L^{2}\left(\Omega_{2}\right)} & =\frac{1}{\sqrt{\lambda_{k} \lambda_{\ell}}}\left(\mathscr{S} \varphi_{k}, \mathscr{S} \varphi_{\ell}\right)_{L^{2}\left(\Omega_{2}\right)}=\frac{1}{\sqrt{\lambda_{k} \lambda_{\ell}}}\left(\mathscr{K} \varphi_{k}, \varphi_{\ell}\right)_{L^{2}\left(\Omega_{1}\right)} \\
& =\frac{\lambda_{k}}{\sqrt{\lambda_{k} \lambda_{\ell}}}\left(\varphi_{k}, \varphi_{\ell}\right)_{L^{2}\left(\Omega_{1}\right)}=\delta_{k, \ell} .
\end{aligned}
$$


If $\lambda_{\ell}=0$ for some $\ell \in \mathbb{N}$, we can extend this collection of functions properly to obtain an orthonormal basis $\left\{\psi_{\ell}\right\}_{\ell \in \mathbb{N}}$ of $L^{2}\left(\Omega_{2}\right)$. Due to

$$
\sqrt{\lambda_{\ell}} \varphi_{\ell}(\mathbf{x})=\frac{1}{\sqrt{\lambda_{\ell}}}\left(\mathscr{S}^{\star} \mathscr{S} \varphi_{\ell}\right)(\mathbf{x})=\left(\mathscr{S}^{\star} \psi_{\ell}\right)(\mathbf{x})=\int_{\Omega_{2}} f(\mathbf{x}, \mathbf{z}) \psi_{\ell}(\mathbf{z}) \mathrm{d} \mathbf{z}
$$

for all $\mathbf{x} \in \Omega_{1}$ and $\ell \in \mathbb{N}$, we finally obtain the representation

$$
f(\mathbf{x}, \mathbf{y})=\sum_{\ell=1}^{\infty} \sqrt{\lambda_{\ell}} \varphi_{\ell}(\mathbf{x}) \psi_{\ell}(\mathbf{y}) .
$$

With (3.4) and (3.5), this equation is easily verified by testing with the orthonormal basis $\left\{\varphi_{k} \otimes \psi_{\ell}\right\}_{k, \ell \in \mathbb{N}}$ of $L^{2}\left(\Omega_{1} \times \Omega_{2}\right)$.

REMARK 3.1 The adjoint kernel $\widetilde{k}(\cdot, \cdot)$ is just obtained by interchanging $\Omega_{1}$ and $\Omega_{2}$, i.e.,

$$
\widetilde{k}\left(\mathbf{y}, \mathbf{y}^{\prime}\right)=\int_{\Omega_{1}} f(\mathbf{x}, \mathbf{y}) f\left(\mathbf{x}, \mathbf{y}^{\prime}\right) \mathrm{d} \mathbf{x} \in L^{2}\left(\Omega_{2} \times \Omega_{2}\right) .
$$

Then, one has the integral operator

$$
\widetilde{\mathscr{K}}=\mathscr{S} \mathscr{S}^{\star}: L^{2}\left(\Omega_{2}\right) \rightarrow L^{2}\left(\Omega_{2}\right), \quad u \mapsto(\widetilde{\mathscr{K}} u)(\mathbf{y}):=\int_{\Omega_{2}} \widetilde{k}\left(\mathbf{y}, \mathbf{y}^{\prime}\right) u\left(\mathbf{y}^{\prime}\right) \mathrm{d} \mathbf{y}^{\prime} .
$$

Again there exists a decomposition into eigenpairs

$$
\widetilde{\mathscr{K}} \widetilde{\varphi}_{\ell}=\tilde{\lambda}_{\ell} \widetilde{\varphi}_{\ell}, \quad \ell \in \mathbb{N},
$$

with non-negative eigenvalues $\tilde{\lambda}_{1} \geqslant \tilde{\lambda}_{2} \geqslant \cdots \geqslant \tilde{\lambda}_{m} \rightarrow 0$ and eigenfunctions $\widetilde{\varphi}_{\ell} \in L^{2}\left(\Omega_{2}\right)$. We also obtain a second sequence of orthonormal functions $\widetilde{\psi}_{\ell} \in L^{2}\left(\Omega_{1}\right)$ analogously to (3.4). The functions $\left\{\widetilde{\varphi}_{\ell}\right\}_{\ell \in \mathbb{N}}$ and $\left\{\widetilde{\psi}_{\ell}\right\}_{\ell \in \mathbb{N}}$ will be the same as before but now their roles are exchanged. Moreover, the eigenvalues $\lambda_{\ell}$ and $\widetilde{\lambda}_{\ell}$ of $\mathscr{K}$ and $\widetilde{\mathscr{K}}$ coincide.

\subsection{Truncation error}

We shall now give improved estimates on the the decay rate of the eigenvalues of the integral operator $\mathscr{K}=\mathscr{S}^{\star} \mathscr{S}$ with kernel (3.3). To this end, assume that $f \in H_{\text {mix }}^{p, 0}\left(\Omega_{1} \times \Omega_{2}\right)$. We introduce new ${ }^{1}$ finite element spaces $U_{M} \subset L^{2}\left(\Omega_{1}\right)$, which consist of $M$ discontinuous, piecewise polynomial functions of total degree $\lceil p\rceil$ on a quasi-uniform triangulation of $\Omega_{1}$ with mesh width $h_{M} \sim M^{-1 / n_{1}}$. Then, due to the Bramble-Hilbert lemma (see e.g., Braess (2001) or Brenner \& Scott (2008)), given a function $w \in H^{p}\left(\Omega_{1}\right)$, the $L^{2}$-orthogonal projection $P_{M}: L^{2}\left(\Omega_{1}\right) \rightarrow U_{M}$ satisfies

$$
\left\|\left(I-P_{M}\right) w\right\|_{L^{2}\left(\Omega_{1}\right)} \leqslant c_{p} M^{-p / n_{1}}\|w\|_{H^{p}\left(\Omega_{1}\right)},
$$

uniformly in $M$. For the approximation of $f(\mathbf{x}, \mathbf{y})$ in the first variable, i.e.

$$
f_{M}(\mathbf{x}, \mathbf{y}):=\left(\left(P_{M} \otimes I\right) f\right)(\mathbf{x}, \mathbf{y}),
$$

we obtain the following approximation result in $U_{M}$, see also Harbrecht et al. (2015).

\footnotetext{
${ }^{1}$ The present argument relies on an approximation argument. The new finite element spaces $\left\{U_{M}\right\}$ are introduced to obtain the optimal convergence rate with $N$ degrees of freedom.
} 
THEOREM 3.1 Let $\lambda_{1} \geqslant \lambda_{2} \geqslant \ldots \geqslant 0$ be the eigenvalues of the operator $\mathscr{K}=\mathscr{S} \mathscr{S}^{\star}$ and $\lambda_{1}^{M} \geqslant \lambda_{2}^{M} \geqslant$ $\ldots \geqslant \lambda_{M}^{M} \geqslant 0$ those of $\mathscr{K}_{M}:=P_{M} \mathscr{K} P_{M}$. Then, it holds

$$
\left\|f-f_{M}\right\|_{L^{2}\left(\Omega_{1} \times \Omega_{2}\right)}^{2}=\operatorname{trace} \mathscr{K}-\operatorname{trace} \mathscr{K}_{M}
$$

and therefore

$$
\left\|f-f_{M}\right\|_{L^{2}\left(\Omega_{1} \times \Omega_{2}\right)}^{2}=\sum_{\ell=1}^{M}\left(\lambda_{\ell}-\lambda_{\ell}^{M}\right)+\sum_{\ell=M+1}^{\infty} \lambda_{\ell} .
$$

Proof. Let $\left\{\theta_{k}\right\}_{k \in \mathbb{N}}$ be an orthonormal basis of $L^{2}\left(\Omega_{1}\right)$ such that either $\theta_{k} \in \operatorname{img} P_{M}$ or $\theta_{k} \in \operatorname{img}\left(I-P_{M}\right)$ holds. This implies $\left(\mathscr{S}\left(I-P_{M}\right) \theta_{k}, \mathscr{S} P_{M} \theta_{k}\right)_{L^{2}\left(\Omega_{2}\right)}=0$ for all $k \in \mathbb{N}$. We thus arrive at

$$
\left\|f-f_{M}\right\|_{L^{2}\left(\Omega_{1} \times \Omega_{2}\right)}^{2}=\int_{\Omega_{2}} \sum_{\ell=1}^{\infty}\left(\left(f-f_{M}\right)(\cdot, \mathbf{y}), \theta_{\ell}\right)_{L^{2}\left(\Omega_{1}\right)}^{2} \mathrm{~d} \mathbf{y} .
$$

Due to the fact that $I-P_{M}$ is an $L^{2}$-orthogonal projection, we have

$$
\left(\left(f-f_{M}\right)(\cdot, \mathbf{y}), \theta_{\ell}\right)_{L^{2}\left(\Omega_{1}\right)}=\left(f(\cdot, \mathbf{y}),\left(I-P_{M}\right) \theta_{\ell}\right)_{L^{2}\left(\Omega_{1}\right)} .
$$

Hence, it holds

$$
\left\|f-f_{M}\right\|_{L^{2}\left(\Omega_{1} \times \Omega_{2}\right)}^{2}=\sum_{\ell=1}^{\infty} \int_{\Omega_{2}}\left(f(\cdot, \mathbf{y}),\left(I-P_{M}\right) \theta_{\ell}\right)_{L^{2}\left(\Omega_{1}\right)}^{2} \mathrm{~d} \mathbf{y} .
$$

Inserting next the definition of $\mathscr{S}$, we obtain

$$
\left\|f-f_{M}\right\|_{L^{2}\left(\Omega_{1} \times \Omega_{2}\right)}^{2}=\sum_{\ell=1}^{\infty}\left\|\mathscr{S}\left(I-P_{M}\right) \theta_{\ell}\right\|_{L^{2}\left(\Omega_{2}\right)}^{2}=\sum_{\ell=1}^{\infty}\left\|\mathscr{S} \theta_{\ell}\right\|_{L^{2}\left(\Omega_{2}\right)}^{2}-\sum_{\ell=1}^{\infty}\left\|\mathscr{S} P_{M} \theta_{\ell}\right\|_{L^{2}\left(\Omega_{2}\right)}^{2}
$$

Finally, by using

$$
\operatorname{trace} \mathscr{K}=\sum_{\ell=1}^{\infty}\left(\mathscr{K} \theta_{\ell}, \theta_{\ell}\right)_{L^{2}\left(\Omega_{1}\right)}=\sum_{\ell=1}^{\infty}\left\|\mathscr{S} \theta_{\ell}\right\|_{L^{2}\left(\Omega_{1}\right)}^{2}
$$

and

$$
\operatorname{trace} \mathscr{K}_{M}=\sum_{\ell=1}^{\infty}\left(\mathscr{K}_{M} \theta_{\ell}, \theta_{\ell}\right)_{L^{2}\left(\Omega_{1}\right)}=\sum_{\ell=1}^{\infty}\left\|\mathscr{S} P_{M} \theta_{\ell}\right\|_{L^{2}\left(\Omega_{1}\right)}^{2},
$$

we conclude the assertion.

By combining this theorem with the approximation estimate (3.7), we can obviously bound the trace error by

$$
0 \leqslant \operatorname{trace} \mathscr{K}-\operatorname{trace} \mathscr{K}_{M} \lesssim M^{-\frac{2 p}{n_{1}}}\|f\|_{H_{\text {mix }}^{p, 0}\left(\Omega_{1} \times \Omega_{2}\right)}^{2} .
$$

This estimate now allows to prove the following result on the truncation of the singular value decomposition after $M$ terms.

TheOREM 3.2 Let $f \in H_{\text {mix }}^{p, 0}\left(\Omega_{1} \times \Omega_{2}\right)$. Then, it holds

$$
\left\|f-\sum_{\ell=0}^{M} \sqrt{\lambda_{\ell}}\left(\varphi_{\ell} \otimes \psi_{\ell}\right)\right\|_{L^{2}\left(\Omega_{1} \times \Omega_{2}\right)} \lesssim M^{-\frac{p}{n_{1}}}\|f\|_{H_{m i x}^{p, 0}\left(\Omega_{1} \times \Omega_{2}\right)} .
$$


Proof. Due to the orthonormality of the sequences $\left\{\varphi_{\ell}\right\}$ and $\left\{\psi_{\ell}\right\}$ in $L^{2}\left(\Omega_{1}\right)$ and $L^{2}\left(\Omega_{2}\right)$, respectively, the error when truncating the singular value decomposition after $M$ terms is given by

$$
\left\|f-\sum_{\ell=0}^{M} \sqrt{\lambda_{\ell}}\left(\varphi_{\ell} \otimes \psi_{\ell}\right)\right\|_{L^{2}\left(\Omega_{1} \times \Omega_{2}\right)}^{2}=\left\|\sum_{\ell=M+1}^{\infty} \sqrt{\lambda_{\ell}}\left(\varphi_{\ell} \otimes \psi_{\ell}\right)\right\|_{L^{2}\left(\Omega_{1} \times \Omega_{2}\right)}^{2}=\sum_{\ell=M+1}^{\infty} \lambda_{\ell} .
$$

In view of Theorem 3.1 and (3.9), since $\lambda_{\ell} \geqslant \lambda_{\ell}^{M}$ for all $\ell \in\{1, \ldots, M\}$ (see e.g. Babuška \& Osborn (1991)), we immediately arrive at the following estimate:

$$
\sum_{\ell=M+1}^{\infty} \lambda_{\ell} \lesssim M^{-\frac{2 p}{n_{1}}}\|f\|_{H_{m i x}^{p, 0}\left(\Omega_{1} \times \Omega_{2}\right)}^{2} .
$$

According to Theorem 3.2, we only need the smoothness of $f$ in the first coordinate to derive estimate (3.10). Since the eigenvalues of integral operator $\mathscr{K}$ and its adjoint $\widetilde{\mathscr{K}}$ are the same, we can also exploit any smoothness of $f$ in the second coordinate, if provided, by interchanging the roles of $\Omega_{1}$ and $\Omega_{2}$ in the above proof. We thus obtain the following corollary.

COROLlaRY 3.1 For $f \in H_{i s o}^{p_{1}, p_{2}}\left(\Omega_{1} \times \Omega_{2}\right)$, it holds

$$
\left\|f-\sum_{\ell=0}^{M} \sqrt{\lambda_{\ell}}\left(\varphi_{\ell} \otimes \psi_{\ell}\right)\right\|_{L^{2}\left(\Omega_{1} \times \Omega_{2}\right)} \lesssim M^{-\max \left\{\frac{p_{1}}{n_{1}}, \frac{p_{2}}{n_{2}}\right\}}\|f\|_{H_{i s o}^{p_{1}, p_{2}}\left(\Omega_{1} \times \Omega_{2}\right)} .
$$

Altogether, in order to ensure the bound

$$
\left\|f-\sum_{\ell=0}^{M} \sqrt{\lambda_{\ell}}\left(\varphi_{\ell} \otimes \psi_{\ell}\right)\right\|_{L^{2}\left(\Omega_{1} \times \Omega_{2}\right)} \lesssim \varepsilon
$$

on the truncation error of the singular value decomposition, we need, as a consequence of Theorem 3.2 and Corollary 3.1, to choose the expansion degree $M$ in accordance with

$$
M \sim \varepsilon^{-\min \left\{\frac{n_{1}}{p_{1}}, \frac{n_{2}}{p_{2}}\right\}}
$$

if $f \in H_{i s o}^{p_{1}, p_{2}}\left(\Omega_{1} \times \Omega_{2}\right)$. Note that in the situation $p=p_{1}=p_{2}$, which means $f \in H^{p}\left(\Omega_{1} \times \Omega_{2}\right)$, the truncation rank is simply given by $M \sim \varepsilon^{-\frac{\min \left\{n_{1}, n_{2}\right\}}{p}}$.

\subsection{Decay of the eigenvalues}

Having Corollary 3.1 at hand, we can give a bound on the decay of the singular values. Note that this estimate improves the bound

$$
\lambda_{\ell} \lesssim \ell^{-2 \min \left\{\frac{p_{1}}{n_{1}}, \frac{p_{2}}{n_{2}}\right\}} \quad \text { as } \ell \rightarrow \infty
$$

from Griebel \& Harbrecht (2014) by an additive factor 1 in the exponent. In particular, the new bound below is now sharp (see, for instance, the specific examples in Griebel \& Harbrecht (2014)). Moreover, it coincides with the bound which was derived in Dölz et al. (2017) and Griebel \& Li (2017) by different techniques. 
Proposition 3.3 Consider $f \in H_{i s o}^{p_{1}, p_{2}}\left(\Omega_{1} \times \Omega_{2}\right)$ with associated kernel $k$ from (3.3) and associated integral operator $\mathscr{K}$ from (3.2). Then, the eigenvalues $\left\{\lambda_{\ell}\right\}_{\ell \in \mathbb{N}}$ of $\mathscr{K}$ decay like

$$
\lambda_{\ell} \lesssim \ell^{-2 \min \left\{\frac{p_{1}}{n_{1}}, \frac{p_{2}}{n_{2}}\right\}-1} \quad \text { as } \ell \rightarrow \infty .
$$

Proof. Since the sequence $\left\{\lambda_{\ell}\right\}$ decreases monotonically, it holds on the one hand

$$
\sum_{m=1}^{2^{k+1}-1} \lambda_{m}=\lambda_{1}+\left(\lambda_{2}+\lambda_{3}\right)+\cdots+\left(\lambda_{2^{k}}+\lambda_{2^{k}+1}+\cdots+\lambda_{2^{k+1}-1}\right) \leqslant \sum_{n=0}^{k} 2^{n} \lambda_{2^{n}}
$$

and on the other hand

$$
\sum_{m=1}^{2^{k}} \lambda_{m}=\lambda_{1}+\lambda_{2}+\left(\lambda_{3}+\lambda_{4}\right)+\cdots+\left(\lambda_{2^{k-1}+1}+\lambda_{2^{k-1}+2}+\cdots+\lambda_{2^{k}}\right) \geqslant \frac{1}{2} \sum_{n=0}^{k} 2^{n} \lambda_{2^{n}} .
$$

This is the well-known Cauchy condensation test, which implies

$$
\sum_{m=2^{k}}^{2^{k+1}-1} \lambda_{m} \sim \sum_{n=0}^{k} 2^{n} \lambda_{2^{n}}
$$

For arbitrary $k \in \mathbb{N}$, in view of (3.11), we conclude with $\beta:=2 \min \left\{\frac{p_{1}}{n_{1}}, \frac{p_{2}}{n_{2}}\right\}$ that

$$
\sum_{n=0}^{k} 2^{n} \lambda_{2^{n}} \sim \sum_{m=2^{k}}^{2^{k+1}} \lambda_{m} \lesssim \sum_{m=2^{k}}^{\infty} \lambda_{m} \lesssim 2^{-\beta k}
$$

This, however, leads to

$$
\sum_{n=0}^{k} 2^{(\beta+1) n} \lambda_{2^{n}} \lesssim 2^{\beta k} \sum_{m=2^{k}}^{2^{k+1}} \lambda_{m} \lesssim 2^{\beta k} \sum_{m=2^{k}}^{\infty} \lambda_{m} \lesssim 1
$$

uniformly in $k \in \mathbb{N}$. Therefore, $2^{(\beta+1) n} \lambda_{2^{n}}$ tends to zero, which immediately implies that $\ell^{\beta+1} \lambda_{\ell}$ also tends to zero since

$$
2^{(\beta+1) n} \lambda_{2^{n}} \sim \ell^{\beta+1} \lambda_{\ell} \sim 2^{(\beta+1)(n+1)} \lambda_{2^{n+1}} \text { for all } 2^{n} \leqslant \ell<2^{n+1} .
$$

\subsection{Numerical approximation}

In Corollary 3.1, we used an exact description of the eigenfunctions. However, this does not hold in practice. Instead, the eigenvalues $\left\{\lambda_{\ell}\right\}_{\ell=1}^{M}$ and eigenfunctions $\left\{\varphi_{\ell}\right\}_{\ell=1}^{M}$ and $\left\{\psi_{\ell}\right\}_{\ell=1}^{M}$ need to be approximately computed in the finite element spaces which have been introduced in Subsection 2.1.

For functions $f \in H_{i s o}^{p_{1}, p_{2}}\left(\Omega_{1} \times \Omega_{2}\right)$ with $p_{1} \leqslant r_{1}$ and $p_{2} \leqslant r_{2}$, we already know that the full tensor product space yields the best possible approximation. Indeed, the singular values then decay not fast enough in order to benefit from additional compression. Hence, we shall assume $p_{1}>r_{1}$ or $p_{2}>r_{2}$ in the subsequent discussion. 
In the following, we first consider the projection of $f$ onto a suitable full tensor product ansatz space and perform afterwards a projection onto $M$ dominant eigenpairs of the projected function.

When choosing the levels of refinement $j_{1}$ and $j_{2}$ for the projections $Q_{j_{1}}^{(1)}$ and $Q_{j_{2}}^{(2)}$ in (2.6), we obtain $N_{\varphi}=\operatorname{dim}\left(V_{j_{1}}^{(1)}\right) \sim 2^{j_{1} n_{1}}$ and $N_{\psi}=\operatorname{dim}\left(V_{j_{2}}^{(2)}\right) \sim 2^{j_{2} n_{2}}$ degrees of freedoms, respectively. Then, for $\mathscr{K}_{N_{\varphi}}:=Q_{j}^{(1)} \mathscr{K} Q_{j}^{(1)}$ with eigenvalues $\lambda_{1, N_{\varphi}} \geqslant \lambda_{2, N_{\varphi}} \geqslant \cdots \geqslant \lambda_{N_{\varphi}, N_{\varphi}} \geqslant 0$, it holds in complete analogy to Theorem 3.1

$$
\begin{aligned}
\left\|\left(I-Q_{j_{1}}^{(1)} \otimes I\right) f\right\|_{L^{2}\left(\Omega_{1} \times \Omega_{2}\right)}^{2} & =\operatorname{trace} \mathscr{K}-\operatorname{trace} \mathscr{K}_{N_{\varphi}} \\
& =\sum_{\ell=1}^{N_{\varphi}}\left(\lambda_{\ell}-\lambda_{\ell, N_{\varphi}}\right)+\sum_{\ell=N_{\varphi}+1}^{\infty} \lambda_{\ell} \\
& \lesssim 2^{-2 j_{1} \min \left\{p_{1}, r_{1}\right\}}\|f\|_{H_{\operatorname{mix}}^{\min \left\{p_{1}, r_{1}\right\}, 0}\left(\Omega_{1} \times \Omega_{2}\right)}^{2}
\end{aligned}
$$

We emphasize again that there holds $\lambda_{\ell} \geqslant \lambda_{\ell, N_{\varphi}}$ for all $\ell \in\left\{1,2, \ldots, N_{\varphi}\right\}$.

For $M \leqslant N_{\varphi}$, let $P_{M}^{(1)}$ denote the projection onto the $M$ dominant eigenpairs $\left(\lambda_{1, N_{\varphi}}, \varphi_{1, N_{\varphi}}\right), \ldots,\left(\lambda_{M, N_{\varphi}}, \varphi_{M, N_{\varphi}}\right)$ of $\mathscr{K}_{N_{\varphi}}$, i.e.,

$$
P_{M}^{(1)}: L^{2}\left(\Omega_{1}\right) \rightarrow L^{2}\left(\Omega_{1}\right), \quad g \mapsto P_{M}^{(1)} g=\sum_{k=1}^{M}\left(g, \varphi_{k, N_{\varphi}}\right)_{L^{2}\left(\Omega_{1}\right)} \varphi_{k, N_{\varphi}}
$$

Then, we have

$$
\begin{aligned}
\|(I- & \left.P_{M}^{(1)} \otimes I\right) f \|_{L^{2}\left(\Omega_{1} \times \Omega_{2}\right)}^{2} \\
& =\operatorname{trace} \mathscr{K}-\operatorname{trace} P_{M}^{(1)} \mathscr{K}_{N_{\varphi}} P_{M}^{(1)} \\
& =\sum_{\ell=1}^{M}\left(\lambda_{\ell}-\lambda_{\ell, N_{\varphi}}\right)+\sum_{\ell=M+1}^{\infty} \lambda_{\ell} \\
& \lesssim 2^{-2 j_{1} \min \left\{p_{1}, r_{1}\right\}}\|f\|_{H_{\text {mix }}^{\min \left\{p_{1}, r_{1}\right\}, 0}\left(\Omega_{1} \times \Omega_{2}\right)}^{2}+M^{-2 \max \left\{\frac{p_{1}}{n_{1}}, \frac{p_{2}}{n_{2}}\right\}}\|f\|_{H_{i s o}^{p_{1}, p_{2}}\left(\Omega_{1} \times \Omega_{2}\right)}^{2},
\end{aligned}
$$

where we used (3.11) and

$$
0 \leqslant \sum_{\ell=1}^{M}\left(\lambda_{\ell}-\lambda_{\ell, N_{\varphi}}\right) \leqslant \sum_{\ell=1}^{N_{\varphi}}\left(\lambda_{\ell}-\lambda_{\ell, N_{\varphi}}\right) \leqslant 2^{-2 j_{1} \min \left\{r_{1}, p_{1}\right\}}\|f\|_{H_{\operatorname{mix}}^{\min \left\{r_{1}, p_{1}\right\}, 0}{ }_{\left(\Omega_{1} \times \Omega_{2}\right)}^{2}}
$$

in accordance with (3.15).

We proceed in complete analogy in the second variable. For the projection

$$
P_{M}^{(2)}: L^{2}\left(\Omega_{2}\right) \rightarrow L^{2}\left(\Omega_{2}\right), \quad g \mapsto P_{M}^{(2)} g=\sum_{k=1}^{M}\left(g, \psi_{k, N_{\psi}}\right)_{L^{2}\left(\Omega_{1}\right)} \psi_{k, N_{\psi}}
$$

onto the $M \leqslant N_{\psi}$ dominant eigenpairs $\left(\widetilde{\lambda}_{1, N_{\psi}}, \psi_{1, N_{\psi}}\right), \ldots,\left(\widetilde{\lambda}_{M, N_{\psi}}, \psi_{M, N_{\psi}}\right)$ of $\widetilde{\mathscr{K}}_{N_{\psi}}:=Q_{j_{2}}^{(2)} \widetilde{\mathscr{K}} Q_{j_{2}}^{(2)}$, we obtain

$$
\begin{aligned}
\|(I-I & \left.\otimes P_{M}^{(2)}\right) f \|_{L^{2}\left(\Omega_{1} \times \Omega_{2}\right)}^{2} \\
& \lesssim 2^{-2 j_{2} \min \left\{p_{2}, r_{2}\right\}}\|f\|_{H_{\text {mix }}^{0, \min \left\{p_{2}, r_{2}\right\}}\left(\Omega_{1} \times \Omega_{2}\right)}^{2}+M^{-2 \min \left\{\frac{p_{1}}{n_{1}}, \frac{p_{2}}{n_{2}}\right\}}\|f\|_{H_{i s o}^{p_{1}, p_{2}}\left(\Omega_{1} \times \Omega_{2}\right)}^{2} .
\end{aligned}
$$


We now choose the levels $j_{1}$ and $j_{2}$ such that (2.7) holds. Then, by combining (3.16) and (3.17), we arrive for any $M \leqslant \max \left\{N_{\varphi}, N_{\psi}\right\}$ at

$$
\begin{aligned}
\|(I- & \left.P_{M}^{(1)} \otimes P_{M}^{(2)}\right) f \|_{L^{2}\left(\Omega_{1} \times \Omega_{2}\right)}^{2} \\
& \lesssim 2^{-2 j_{1} \min \left\{p_{1}, r_{1}\right\}}\|f\|_{H_{\operatorname{mix}}^{\min \left\{p_{1}, r_{1}\right\}, 0}\left(\Omega_{1} \times \Omega_{2}\right)}^{2}+M^{-2 \min \left\{\frac{p_{1}}{n_{1}}, \frac{p_{2}}{n_{2}}\right\}}\|f\|_{H_{i s o}^{p_{1}, p_{2}}\left(\Omega_{1} \times \Omega_{2}\right)}^{2} .
\end{aligned}
$$

Note that the expression $\left(P_{M}^{(1)} \otimes P_{M}^{(2)}\right) f$ corresponds to the desired series expansion

$$
\left(P_{M}^{(1)} \otimes P_{M}^{(2)}\right) f=\sum_{\ell=1}^{M} \alpha_{\ell}\left(\varphi_{\ell, N_{\varphi}} \otimes \psi_{\ell, N_{\psi}}\right),
$$

where the coefficients $\left\{\alpha_{\ell}\right\}$ are given by

$$
\alpha_{\ell}:=\left(f, \varphi_{\ell, N_{\varphi}} \otimes \psi_{\ell, N_{\psi}}\right)_{L^{2}\left(\Omega_{1} \times \Omega_{2}\right)} \quad \text { for all } \ell \in\{1,2, \ldots, M\} .
$$

The approximation error is already fixed by the projection onto the full tensor product space. Hence, it remains to equilibrate the truncation error, which is induced by $M$, and the projection error, which is related to the degrees of freedom $N_{\varphi}$ and $N_{\psi}$, respectively. This implies the choice

$$
M \sim \varepsilon^{-\min \left\{\frac{n_{1}}{p_{1}}, \frac{n_{2}}{p_{2}}\right\}}, \quad N_{\varphi} \sim \varepsilon^{-\frac{n_{1}}{\min \left\{p_{1}, r_{1}\right\}}}, \quad N_{\psi} \sim \varepsilon^{-\frac{n_{2}}{\min \left\{p_{2}, r_{2}\right\}}} .
$$

In particular, the assumption $p_{1}>r_{1}$ or $p_{2}>r_{2}$ implies both, $M \leqslant N_{\varphi}$ and $M \leqslant N_{\psi}$. Thus, in view of the cost $M \cdot \max \left\{N_{\varphi}, N_{\psi}\right\}$, we obtain the following result:

THEOREM 3.4 The number of degrees of freedom, which is needed to approximate a function $f \in$ $H_{i s o}^{p_{1}, p_{2}}\left(\Omega_{1} \times \Omega_{2}\right)$ by the singular value decomposition approach (3.1) to a prescribed accuracy $\varepsilon$, is

$$
\operatorname{dof}_{s v d}(\varepsilon) \sim \varepsilon^{-\min \left\{\frac{n_{1}}{p_{1}}, \frac{n_{2}}{p_{2}}\right\}} \varepsilon^{-\max \left\{\frac{n_{1}}{\min \left\{p_{1}, r_{1}\right\}}, \frac{n_{2}}{\min \left\{p_{2}, r_{2}\right\}}\right\}} .
$$

REMARK 3.2 Note that if $p_{1} \leqslant r_{1}$ and $p_{2} \leqslant r_{2}$, the cost complexity (3.18) also covers the situation when the full tensor product space coincides with Kolmogorov's $n$-width by means of $M=\min \left\{N_{\varphi}, N_{\psi}\right\}$. We may hence drop the restriction $p_{1}>r_{1}$ or $p_{2}>r_{2}$ which has been made in the beginning of this subsection. Moreover, the cost complexity (3.18) does not improve if $f$ would possess mixed Sobolev regularity in the sense of $f \in H_{\text {mix }}^{p_{1}, p_{2}}\left(\Omega_{1} \times \Omega_{2}\right)$.

We emphasize that the estimate (3.18) does not include the work to be spent for computing the singular values nor the eigenfunctions. Here, a naive approach would result in a cost complexity of order $M \cdot N^{2}$, where $N:=\max \left\{N_{\varphi}, N_{\psi}\right\}$, the use of fast methods for nonlocal operators would result in an almost linear or even linear complexity per eigenpair. Note that, in any case, at least linear complexity $\mathscr{O}(M \cdot N)$ is required, which is indeed achieved with modern algorithms, see e.g. Dahmen et al. (2008), Dai et al. (2008), and the references cited therein. Therefore, our analysis is based on the best possible situation for the approximative truncated singular value decomposition and our later comparison will be fair in this respect.

REMARK 3.3 In uncertainty quantification, the truncated singular value decomposition of the random field under consideration is called Karhunen-Loéve approximation. In particular, one has then higher regularity only in the spatial variable, i.e., one considers functions $f \in H_{\text {mix }}^{0, p}\left(\Omega_{1} \times \Omega_{2}\right)$, where $\Omega_{1}$ reflects 
the sample space and $\Omega_{2}$ the physical domain. Of course, the truncation estimate (3.13) remains true with $p_{1}=0$ and $p_{2}=p$, but an approximation of the eigenfunctions with respect to the sample space is not possible any more. Instead, one prescribes a certain distribution of the random variables which involves a modelling step, see Ghanem \& Spanos (1991) or Le Maître \& Knio (2010) for details.

\section{Sparse grids}

Based on the multiscale analyses (2.5) on each individual subdomain, one naturally obtains a second method to approximate functions in tensor product spaces: By choosing complementary spaces

$$
W_{j}^{(i)}=\operatorname{span}\left\{\xi_{j, k}^{(i)}: k \in \nabla_{j}^{(i)}:=\Delta_{j}^{(i)} \backslash \Delta_{j-1}^{(i)}\right\}, \quad i=1,2,
$$

such that

$$
V_{j}^{(i)}=W_{j}^{(i)} \oplus V_{j-1}^{(i)}, \quad V_{0}^{(i)}=W_{0}^{(i)},
$$

we can define the so called general sparse grid space, see Bungartz \& Griebel (2004) and Griebel \& Harbrecht (2013),

$$
\widehat{\mathbf{V}}_{J}^{\sigma}:=\bigoplus_{j_{1} \sigma+j_{2} / \sigma \leqslant J} W_{j_{1}}^{(1)} \otimes W_{j_{2}}^{(2)}
$$

where $\sigma>0$ is a given parameter. Thus, a function $\widehat{f}_{J} \in \widehat{\mathbf{V}}_{J}^{\sigma}$ is represented as

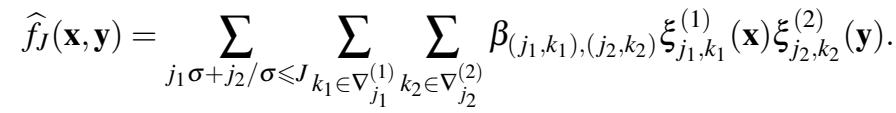

Sparse grids can be constructed via hierarchical bases, interpolets and wavelet-like bases (see e.g. DeVore et al. (1998), Griebel \& Knapek (2009), Strömberg (1998), and Zenger (1991)) or even directly by finite elements in terms of frames (see e.g. Griebel (1994), Griebel \& Oswald (1994), and Harbrecht et al. (2008b)). They can also be build from Fourier-, Chebyshev-, Legendre- or similar global polynomial systems, depending on the respective situation. This results in the so-called hyperbolic cross methods. For a survey on sparse grids, we refer the reader to Bungartz \& Griebel (2004) and the references therein.

The dimension of the general sparse grid space $\widehat{\mathbf{V}}_{J}^{\sigma}$ is essentially equal to the dimension of the finest univariate finite element spaces which enter its construction, i.e., it is essentially equal to the value of $\max \left\{\operatorname{dim} V_{J / \sigma}^{(1)}, \operatorname{dim} V_{J \sigma}^{(2)}\right\}$. Nevertheless, by considering smoothness in terms of mixed Sobolev spaces, its approximation power is essentially the same as in the full tensor product space. Precisely, in accordance with Griebel \& Harbrecht (2013), we have the following result:

LEMMA 4.1 The sparse grid space $\widehat{\mathbf{V}}_{J}^{\sigma}$ possesses

$$
\operatorname{dim} \widehat{\mathbf{V}}_{J}^{\sigma} \sim \begin{cases}2^{J \max \left\{n_{1} / \sigma, n_{2} \sigma\right\}}, & \text { if } n_{1} / \sigma \neq n_{2} \sigma \\ 2^{J n_{2} \sigma} J, & \text { if } n_{1} / \sigma=n_{2} \sigma\end{cases}
$$

degrees of freedom. Moreover, for a given function $f \in H_{\text {mix }}^{p_{1}, p_{2}}\left(\Omega_{1} \times \Omega_{2}\right)$ with $0<p_{1} \leqslant r_{1}$ and $0<$ $p_{2} \leqslant r_{2}$, there holds the approximation estimate

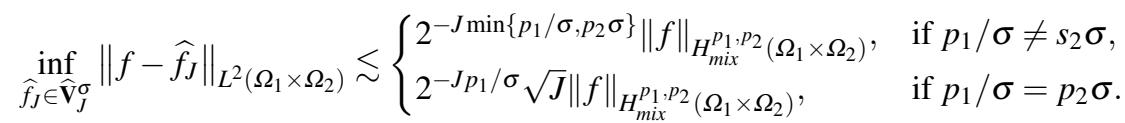


As shown in Griebel \& Harbrecht (2013), the best cost complexity is obtained for the choice $\sigma=$ $\sqrt{n_{1} / n_{2}}$ which means that the degrees of freedom in the extremal spaces $V_{J / \sigma}^{(1)}$ and $V_{J \sigma}^{(2)}$ are equilibrated. It especially holds:

THEOREM 4.1 The number of degrees of freedom, which is needed to approximate a function $f \in$ $H_{\text {mix }}^{p_{1}, p_{2}}\left(\Omega_{1} \times \Omega_{2}\right)$ in the sparse grid space $\widehat{\mathbf{V}}_{J}^{\sigma}$ with $\sigma=\sqrt{n_{1} / n_{2}}$ to a prescribed accuracy $\varepsilon$, is essentially

$$
\operatorname{dof}_{s g}^{\text {mix }}(\varepsilon) \sim \varepsilon^{-\max \left\{\frac{n_{1}}{\min \left\{p_{1}, r_{1}\right\}}, \frac{n_{2}}{\min \left\{p_{2}, r_{2}\right\}}\right\}}
$$

In Theorem 4.1, the convergence rate is given for a function $f \in H_{m i x}^{p_{1}, p_{2}}\left(\Omega_{1} \times \Omega_{2}\right)$. Nonetheless, we are also interested in the convergence rate if the smoothness of $f$ is measured in the isotropic Sobolev space $H_{i s o}^{p_{1}, p_{2}}\left(\Omega_{1} \times \Omega_{2}\right)$. Since for all $q_{1}=p_{1} s$ and $q_{2}=p_{2}(1-s)$ with $s \in[0,1]$ it holds

$$
H_{\text {mix }}^{q_{1}, q_{2}}\left(\Omega_{1} \times \Omega_{2}\right) \subset H_{i s o}^{p_{1}, p_{2}}\left(\Omega_{1} \times \Omega_{2}\right),
$$

we conclude in view of (4.3) the cost complexity

$$
\operatorname{dof}_{s g}^{i s o}(\varepsilon) \sim \min _{0<s<1} \varepsilon^{-\max \left\{\frac{n_{1}}{\min \left\{s p_{1}, r_{1}\right\}}, \frac{n_{2}}{\min \left\{(1-s) p_{2}, r_{2}\right\}}\right\}}
$$

for functions in $H_{i s o}^{p_{1}, p_{2}}\left(\Omega_{1} \times \Omega_{2}\right)$.

The optimum $s$ is the one that equilibrates the fractions $\frac{n_{1}}{s p_{1}}$ and $\frac{n_{2}}{(1-s) p_{2}}$, which yields

$$
\operatorname{dof}_{s g}^{i s o}(\varepsilon) \sim \varepsilon^{-\max \left\{\frac{n_{1}}{p_{1}}+\frac{n_{2}}{p_{2}}, \frac{n_{1}}{r_{1}}, \frac{n_{2}}{r_{2}}\right\}} .
$$

We may hence summarize:

THEOREM 4.2 The number of degrees of freedom, which is needed to approximate a function $f \in$ $H_{i s o}^{p_{1}, p_{2}}\left(\Omega_{1} \times \Omega_{2}\right)$ in the sparse grid space $\widehat{\mathbf{V}}_{J}^{\sigma}$ with $\sigma=\sqrt{n_{1} / n_{2}}$ to a prescribed accuracy $\varepsilon$, is essentially (4.4).

\section{Discussion and concluding remarks}

In the present article, we derived the cost complexities of the approximative truncated singular value decomposition and the general sparse grid approach for given, fixed approximation orders $r_{1}$ and $r_{2}$ and spatial dimensions $n_{1}$ and $n_{2}$ of the associated finite element methods. We are aware that this setting is different to the one in approximation theory, but it clearly reflects practical needs. For making a decision on which method is preferable, we will compare the two different approximation schemes in case of a bivariate function $f \in H_{i s o}^{p_{1}, p_{2}}\left(\Omega_{1} \times \Omega_{2}\right)$.

According to Theorems 3.4 and 4.2 , the cost complexity to ensure a desired approximation error $\varepsilon$ is $\mathscr{O}\left(\varepsilon^{-q}\right)$ with

$$
q_{s v d}=\min \left\{\frac{n_{1}}{p_{1}}, \frac{n_{2}}{p_{2}}\right\}+\max \left\{\frac{n_{1}}{\min \left\{p_{1}, r_{1}\right\}}, \frac{n_{2}}{\min \left\{p_{2}, r_{2}\right\}}\right\}
$$

for the approximation by the singular value decomposition and with

$$
q_{s g}=\max \left\{\frac{n_{1}}{p_{1}}+\frac{n_{2}}{p_{2}}, \frac{n_{1}}{r_{1}}, \frac{n_{2}}{r_{2}}\right\}
$$


for the sparse grid method.

Straightforward calculation shows that $q_{s v d} \geqslant q_{s g}$ for all choices of $n_{1}, n_{2}, r_{1}, r_{2}, p_{1}, p_{2}$. If $p_{1}>r_{1}$ and $p_{2}>r_{2}$, then it even holds $q_{s v d}>q_{s g}$. This means that the approximation by the properly balanced spars grids is superior over the approximation by the singular value decomposition in the function class $H_{i s o}^{p_{1}, p_{2}}\left(\Omega_{1} \times \Omega_{2}\right)$. We emphasize that a change from the isotropic Sobolev space $H_{i s o}^{p_{1}, p_{2}}\left(\Omega_{1} \times \Omega_{2}\right)$ to the anisotropic Sobolev space $H_{m i x}^{p_{1}, p_{2}}\left(\Omega_{1} \times \Omega_{2}\right)$ does only improve the rate $q_{s g}$ in accordance with

$$
q_{s g}=\max \left\{\frac{n_{1}}{\min \left\{p_{1}, r_{1}\right\}}, \frac{n_{2}}{\min \left\{p_{2}, r_{2}\right\}}\right\}
$$

while $q_{s v d}$ is kept unchanged.

In the situation of low regularity, that is, if $p_{1} \leqslant r_{1}$ and $p_{2} \leqslant r_{2}$, the approximative truncated singular value decomposition coincides with the approximation in the full tensor product space $V_{j_{1}}^{(1)} \otimes V_{j_{2}}^{(2)}$, where $j_{1}$ and $j_{2}$ are related by (2.7). This full tensor product space is known to realize Kolmogorov's $n$-width $\varepsilon^{-\frac{n_{1}}{p_{1}}-\frac{n_{2}}{p_{2}}}$ for Sobolev balls in the space $H_{i s o}^{p_{1}, p_{2}}\left(\Omega_{1} \times \Omega_{2}\right)$. The sparse grid approach would essentially also give Kolmogorov's $n$-width, but has in general larger constants.

In case of the sparse grid approach, we envision further improvements by the use of local adaptivity, which would further increase its performance.

In case of the singular value decomposition, the truncation length is determined by the smoothness of the function under consideration and is thus fixed. Therefore, improvements for the truncated singular value decomposition can only be achieved by a more efficient representation of the eigenfunctions.

Future work is needed to study the cost complexity in the higher dimensional setting. Especially, the comparison of the recursive application of the singular value decomposition in a hierarchical tensor format and the sparse grid approach would be relevant for practical application.

Acknowledgement. This research was done while M. Griebel held a John von Neumann Visiting Professorship at the Technische Universität München in the summer term 2017. The hospitality of the Fakultät für Mathematik of the Technische Universität München and especially of the host, Prof. Dr. Folkmar Bornemann, is greatfully acknowledged. H. Harbrecht acknowledges the support by the Swiss National Science Foundation (SNSF) through the project " $\mathscr{H}$ - matrix based first and second moment analysis" (grant 200021_156101).

\section{References}

BABUŠKA, I. (1968) Über universal optimale Quadraturformeln. Appl. Mat., 13, 304-338, 338-404.

BABUŠKA, I. \& OsBorn, J. (1991) Eigenvalue Problems. In Handbook of Numerical Analysis, vol. II, North-Holland, Amsterdam, pages 641-784.

BALESCU, R. (1997) Statistical Dynamics, Matter out of Equilibrium. Imperial College Press, Imperial College, London.

Bebendorf, M. (2011) Adaptive cross approximation of multivariate functions. Constr. Approx., 34, 149-179.

Braess, D. (2001) Finite Elements. Theory, Fast Solvers, and Applications in Solid Mechanics. Cambridge University Press, Cambridge. 
Brenner, S. \& ScotT, L. (2008) The Mathematical Theory of Finite Element Methods. Springer, Berlin.

Bungartz, H.J. \& Griebel, M. (2004) Sparse grids. Acta Numerica, 13, 147-269.

Cioranescu, D. \& Damlamian, A. \& Griso, G. (2008) The periodic unfolding method in homogenization. SIAM J. Appl. Math., 40, 1585-1620.

Dahmen, W. \& Rohwedder, T. \& Schneider, R. \& Zeiser, A. (2008) Adaptive eigenvalue computation: complexity estimates. Numer. Math., 110, 277-312.

DAI, X. \& XU, J. \& ZHOU, A. (2008) Convergence and optimal complexity of adaptive finite element eigenvalue computations. Numer. Math., 110, 313-355.

DEB, M.K. \& BABUŠKA, I. \& OdEN, J.T. (2001) Solution of stochastic partial differential equations using Galerkin finite element techniques. Comput. Methods Appl. Mech. Engrg., 190(48), 6359-6372.

DeVore, R. \& Konyagin, S. \& Temlyakov, V. (1998) Hyperbolic wavelet approximation. Constr. Approx., 14, 1-26.

Dölz, J. \& Harbrecht, H. \& Schwab, C. (2017) Covariance regularity and $\mathscr{H}$-matrix approximation for rough random fields. Numer. Math., 135, 1045-1071.

Ghanem, R.G. \& Spanos, P.D. (1991) Stochastic Finite Elements: A Spectral Approach. Springer, New York.

GrASEDYCK, L. (2010) Hierarchical singular value decomposition of tensors. SIAM J. Matrix Anal. Appl., 31, 2029-2054.

GRIEBEL, M. (1994) Multilevelmethoden als Iterationsverfahren über Erzeugendensystemen. Teubner Skripten zur Numerik. B.G. Teubner, Stuttgart.

Griebel, M. \& HARbrecht, H. (2013). On the construction of sparse tensor product spaces. Math. Comput., 82, 975-994.

Griebel, M. \& Harbrecht, H. (2014). Approximation of bi-variate functions. Singular value decomposition versus sparse grids. IMA J. Numer. Anal., 34, 28-54.

Griebel, M. \& KNAPEK, S. (2009). Optimized general sparse grid approximation spaces for operator equations. Math. Comput., 78, 2223-2257.

GRIEBEL, M. \& LI, G. (2017). On the decay rate of the singular values of bivariate functions. INS Preprint 1702, Institute for Numerical Simulation, University of Bonn.

Griebel, M. \& Oeltz, D. (2007). A sparse grid space-time discretization scheme for parabolic problems. Computing, 81, 1-34.

Griebel, M. \& Oswald, P. (1994). On additive Schwarz preconditioners for sparse grid discretizations. Numer. Math., 66, 449-463.

Hackbusch, W. (2012). Tensor Spaces and Numerical Tensor Calculus. Springer, Berlin-Heidelberg. 
Hackbusch, W. \& KÜHn, S. (2009). A new scheme for the tensor representation. J. Fourier Anal. Appl., 15(5), 706-722.

Harbrecht, H. \& SChNEIDER, R. \& Schwab, C. (2008) Multilevel frames for sparse tensor product spaces. Numer. Math., 110, 199-220.

Harbrecht, H. \& Peters, M. \& Siebenmorgen, M. (2015) Efficient approximation of random fields for numerical applications. Numer. Linear Algebra Appl., 22, 596-617.

Hesthaven, J.S. \& Rozza, G. \& Stamm, B. (2016) Certified Reduced Basis Methods for Parametrized Partial Differential Equations. SpringerBriefs in Mathematics. Springer, Cham.

Kolmogorov, A. (1936) Über die beste Annäherung von Funktionen einer gegebenen Funktionenklasse. Annals Math., 37, 107-110.

Le Maître, O.P. \& KNIO, O.M. (2010) Spectral Methods for Uncertainty Quantification. With Applications to Computational Fluid Dynamics. Scientific Computation. Springer, Dordrecht-HeidelbergLondon-New York.

Lòeve, M. (1978) Probability Theory, Vol. I+II, Springer, New York.

NovaK, E. \& WoźniakowsKi, H. (2008) Tractability of Multivariate Problems: Linear Information. EMS Tracts in Mathematics, European Mathematical Society, Zürich, 2008.

Novak, E. \& Woźniakows KI, H. (2010) Tractability of Multivariate Problems: Standard Information for Functionals. EMS Tracts in Mathematics, European Mathematical Society, Zürich, 2010.

NovaK, E. \& WoŹNiAKowsKi, H. (2012) Tractability of Multivariate Problems: Standard Information for Operators. EMS Tracts in Mathematics, European Mathematical Society, Zürich, 2012.

Oseledets, I.V. \& TyRTyshnikov, E.E. (2009) Breaking the curse of dimensionality, or how to use SVD in many dimensions. SIAM J. Sci. Comput., 31(5), 3744-3759.

Quarteroni, A. \& Manzoni A. \& Negri, F.(2016) Reduced Basis Methods for Partial Differential Equations. An Introduction. Springer, Cham.

RozzA, G. \& HUYnh, D.B.P. \& PATERA, A.T. (2008) Reduced basis approximation and a posteriori error estimation for affinely parametrized elliptic coercive partial differential equations. Application to Transport and Continuum Mechanics. Arch. Comput. Methods Eng., 15, 229-275.

Strömberg, J. (1998) Computation with wavelets in higher dimensions. In Proceedings of the International Congress of Mathematicians, Vol. III, Berlin, 1998, Doc. Math., Extra Vol. III, 523532.

Widmer, G. \& Hiptmair, R. \& Schwab, C. (2008) Sparse adaptive finite elements for radiative transfer. J. Comp. Phys., 227(12), 6071-6105.

Motornys, V. (1974) On the best quadrature formula of the form $\sum_{k=1}^{n} p_{k} f\left(x_{k}\right)$ for some classes of periodic differentiable functions. Ivz. Akad. Nauk USSR Ser. Mat., 38, 538-614.

ZENGER, C. (1991) Sparse grids. In Parallel algorithms for partial differential equations, Proceedings of the 6th GAMM-Seminar, Kiel/Germany, 1990, Notes Numer. Fluid Mech. 31, pages 241-251, Vieweg, Braunschweig. 


\section{LATEST PREPRINTS}

No. Author: Title

2016-18Ｒ. N. Gantner, M. D. Peters

Higher order quasi-Monte Carlo for Bayesian shape inversion

2016-19 C. Urech

Remarks on the degree growth of birational transformations

2016-20 S. Dahlke, H. Harbrecht, M. Utzinger, M. Weimar

Adaptive wavelet BEM for boundary integral equations: Theory and numerical experiments

2016-21 A. Hyder, S. Iula, L. Martinazzi

Large blow-up sets for the prescribed Q-curvature equation in the Euclidean space

2016-22 P. Habegger

The norm of Gaussian periods

2016-23 P. Habegger

Diophantine approximations on definable sets

2016-24 F. Amoroso, D. Masser

Lower bounds for the height in Galois extensions

2016-25 W. D. Brownawell, D. W. Masser

Zero estimates with moving targets

2016-26 H. Derksen, D. Masser

Linear equations over multiplicative groups, recurrences, and mixing III

2016-27Ｉ. Bertrand, D. Masser, A. Pillay, U. Zannier

Relative Manin-Mumford for semi-abelian surfaces

2016-28Ｉ. Capuano, D. Masser, J. Pila, U. Zannier

Rational points on Grassmannians and unlikely intersections in tori

2016-29 C. Nobili, F. Otto

Limitations of the background field method applied to Rayleigh-Bénard convection

2016-30 W. D. Brownawell, D. W. Masser

Unlikely intersections for curves in additive groups over positive characteristic

2016-31 M. Dambrine, H. Harbrecht, M. D. Peters, B. Puig

On Bernoulli's free boundary problem with a random boundary

Preprints are available under https://math.unibas.ch/research/publications 


\section{LATEST PREPRINTS}

No.

2016-32

2016-33

2016-34

2017-01

2017-02

2017-04

2017-05

2017-06

2017-07

2017-08
Author: Title

H. Harbrecht, J. Tausch

A fast sparse grid based space-time boundary element method for the nonstationary heat equation

S. Iula

A note on the Moser-Trudinger inequality in Sobolev-Slobodeckij spaces in dimension one

\section{Bürli, H. Harbrecht, P. Odermatt, S. Sayasone, N. Chitnis}

Mathematical analysis of the transmission dynamics of the liver fluke, Opisthorchis viverrini

\section{J. Dölz and T. Gerig, M. Lüthi, H. Harbrecht and T. Vetter}

Efficient computation of low-rank Gaussian process models for surface and image registration

M. J. Grote, M. Mehlin, S. A. Sauter

Convergence analysis of energy conserving explicit local time-stepping methods for the wave equation

Y. Bilu, F. Luca, D. Masser

Collinear CM-points

P. Zaspel

Ensemble Kalman filters for reliability estimation in perfusion inference

J. Dölz and H. Harbrecht

Hierarchical Matrix Approximation for the Uncertainty Quantification of

Potentials on Random Domains

P. Zaspel

Analysis and parallelization strategies for Ruge-Stüben AMG on many-core processors

\section{H. Harbrecht and M. Schmidlin}

Multilevel Methods for Uncertainty Quantification of Elliptic PDEs with Random Anisotropic Diffusion

M. Griebel and H. Harbrecht

Singular value decomposition versus sparse grids: Refined complexity estimates 